\title{
Necesidad de incluir la política ambiental en los diseños curriculares universitarios
}

\section{Need to include environmental policy in university curriculum designs}

Irma Rosario Estrada Ortiz

Maestría en Docencia Universitaria

Universidad de San Carlos de Guatemala

trabajofeliz2009@hotmail.com

https://orcid.org/0000-0002-4399-0556

Recibido 25/04/2021

Aceptado 15/07/2021

\section{Referencia}

Estrada Ortiz, I. R. (2021). Necesidad de incluir la política ambiental en los diseños curriculares universitarios, Revista Docencia Universitaria, 2(2), 1-9. https://doi.org/10.46954/revistadusac.v2i2.26

\section{Resumen}

El presente artículo tiene su origen en el trabajo de tesis "La observancia de la Política Ambiental en la Educación Universitaria", el cual, en la actualidad se está desarrollando, a fin de poder establecer si la Política Ambiental de la Universidad de San Carlos de Guatemala, la cual fue aprobada en fecha 30 de junio 2014 mediante acta 13-2014, punto sexto, inciso 6.2 por el Consejo Superior Universitario, se encuentra incluida en el Diseño Curricular de la Licenciatura en Psicología, ya que, se considera indispensable incluirla con lo cual se busca una formación holística de los estudiantes de psicología. Aunque la temática es ampliamente conocida en el nivel universitario, no es lo mismo conocerla que contemplarla para orientar su aplicación. En el trabajo de tesis realizado se utilizó un enfoque mixto, un diseño fenomenológico, una muestra no probabilística, se tuvo a la vista la documentación de mérito, como lo es, el Diseño Curricular, de la Licenciatura de Psicología y se trabajó una tabla de cotejo. Posteriormente se realizó el análisis 
Palabras clave:

diseño curricular, política ambiental de la USAC, educación superior, estudiantes.
Keywords:

curriculum design, USAC environmental policy, higher education, students. y discusión de resultados. Los resultados evidencian que en el Diseño Curricular objeto de estudio, se contempla de manera muy vaga la Política Ambiental de la USAC, toda vez que, no aparece en su Misión. Se concluye que se debe incluir la Política Ambiental mencionada, con el objetivo de alcanzar la formación integral de los educandos y un perfil de egreso en el cual los estudiantes hayan adquirido las competencias necesarias sobre el medio ambiente para beneficio de su comunidad.

\section{Abstract}

This article originates from the thesis work "Observance of Environmental Policy in University Education", which is currently being developed, in order to establish whether the Environmental Policy of the University of San Carlos de Guatemala, which was approved on June 30,2014 by act 13-2014, point six, paragraph 6.2 by the University Higher Council, is included in the Curriculum Design of the Bachelor of Psychology, since, it is considered essential to include it with which a holistic training of psychology students is sought. Although the theme is widely known at the university level, it is not the same to know it as to contemplate it to guide its application. In the thesis work carried out a mixed approach was used, a phenomenological design, a nonprobabilistic sample, the merit documentation, as it is, the Curriculum Design, of the Bachelor of Psychology was worked and a comparison table was worked. Subsequently, the analysis and discussion of results was carried out. The results show that in the Curriculum Design under study, USAC's Environmental Policy is very vaguely contemplated, since it does not appear in its Mission. It is concluded that the aforementioned Environmental Policy should be included, with the aim of achieving the comprehensive training of students and an egress profile in which students have acquired the necessary competences on the environment for the benefit of their community.

\section{Introducción}

La elaboración de un Diseño Curricular, responde precisamente a la necesidad de incluir en el mismo, un perfil de egreso de competencias que deben ser alcanzadas por los estudiantes durante su formación como profesionales de la Psicología. El tema del medio ambiente, es de interés a nivel mundial y la educación universitaria no puede desarrollarse al margen del mismo, tomando en cuenta que, a futuro, de nada servirá tener un cúmulo de conocimientos y tecnología de primera línea, si no se tiene un lugar seguro para vivir. El estudio se 
lleva a cabo a raíz del interés por conocer las competencias alcanzadas por los educandos de la licenciatura en psicología al egresar del CUNSAC, con relación al medio ambiente y si éstos poseían o no el perfil necesario sobre dicho tema.

Es una investigación sumamente importante, para el área del conocimiento, porque, mientras se ignore, qué es el medio ambiente y por qué es importante no se logrará concientizar a la población y estimularla a que trabaje voluntariamente a favor del medio ambiente. El estudio tiene como objetivo establecer si se observa la Política Ambiental de la USAC en la docencia universitaria; y uno de los objetivos específicos, sí esa Política Ambiental se incluye en el Diseño Curricular de la Licenciatura de Psicología.

Como una respuesta a la urgente demanda del cuidado del medio ambiente la Universidad de San Carlos de Guatemala, implementó la Política Ambiental, de conservación del medio ambiente, la cual, fue aprobada por el Consejo Superior Universitario, durante la gestión como Rector Magnífico del Dr. Carlos Guillermo Alvarado Cerezo.

En la revista científica Azul, Hernández, Damaris, señala que, según Riojas (citado por Núñez, 2004) este plantea 3 temas principales para concientizar el tema del medio ambiente en la educación universitaria señalando 2 en pregrado y la inclusión de cursos con enfoques ambientales en los programas de estudio e incluir lo relacionado al medio ambiente dentro del diseño curricular. Señala que en Cuba ha sido una estrategia curricular que a través de todo el diseño se incluyan cursos con contenidos de formación ambiental ya que esta educación no puede estar restringida por su importancia y alcance a una asignatura o disciplina.

De lo expuesto se evidencia, la urgencia de incluir en los Diseños Curriculares cursos sobre temas ambientales. Ya no se trata solamente de concientizar a los estudiantes a cerca del respeto y cuidado del medio ambiente, sino motivar también la investigación sobre calentamiento global, tala de árboles, contaminación del aire y del agua, contaminación visual y contaminación auditiva y propiciar la elaboración y desarrollo de proyectos en pro del medio ambiente en sus propias comunidades. Se deben priorizar entre los estudiantes, actividades extramuros que tiendan a educar a la sociedad en cuanto al cuidado, respeto y mantenimiento del medio ambiente. 
Fernández, F., y Moreno, Ma., en suartículocientíficoseñalanla necesidad de plantearse el currículo de forma que sirva no sólo a los objetivos de aprendizaje científico, sino también a los de actitudes y valores y responsabilidad, propios de la Educación Ambiental, expresados en la Carta de Belgrado, y necesarios en la sociedad democrática Charles (1982), esto llevo a rediseñar y a introducir una metodología a la que denominaron "Método de Proyectos de Investigación", al estar basado en la metodología de proyectos coherente con los objetivos vinculados con la Educación Ambiental.

\section{Enfoque, diseño y alcance de la investigación}

La investigación incluye un enfoque mixto, con un diseño fenomenológico, el plan de investigación aún, cuando es un tema que podría abordarse a nivel nacional, fue desarrollado específicamente en San Antonio Aguas Calientes, Municipio de Sacatepéquez, del Departamento de Guatemala, en el Centro Universitario de la USAC, en conjunto con las Autoridades Administrativas del Centro, con los docentes de la Licenciatura en Psicología, y con los estudiantes del segundo, cuarto, sexto y octavo semestre 2020, de julio 2020 al 30 de junio 2021. Siendo el tema principal la observancia de la Política Ambiental de la USAC, en la Licenciatura de Psicología, tema relevante tomando en cuenta las consecuencias que trae el descuido al medio ambiente a nivel global.

\section{Materiales y Métodos}

La investigación en general incluye un proceso de recopilación de datos mediante un enfoque mixto, utilizándose como instrumentos, entrevista semiestructurada a las Autoridades del Centro Universitario, a los docentes y una encuesta a los estudiantes. El presente artículo se refiere a uno de los objetivos de la investigación, que incluye el análisis de documentos, mediante una tabla de cotejo, aplicada al Diseño Curricular de la Licenciatura en Psicología. Se utilizó una muestra no probabilística, con ocho docentes y 16 estudiantes de la Licenciatura en Psicología, de los semestres segundo, cuarto, sexto y octavo; y, se lleva acabo, una triangulación para la obtención de resultados fidedignos. 


\section{Resultados}

A través del análisis del Diseño Curricular de la Licenciatura en Psicología, se establece que la Política Ambiental de la USAC, no se encuentra presente dentro del mismo, así como tampoco se ve reflejada en la Misión y Visión de dicho Diseño, ni en el perfil de egreso de los estudiantes, así como tampoco en los programas de estudio, en donde solamente en el 9o. semestre aparecen como cursos optativos, Psicología Ambiental y Ecología. Según lo establecido en la Política Ambiental, las 40 unidades académicas deben contemplar la mencionada Política, tanto en su Diseño Curricular como en sus programas de estudio, proceso a cargo de la Dirección General de Docencia -DIGED- por medio de la cual se canalizaría la información a todas las Autoridades de las Facultades, Escuelas y Centros Regionales.

En la tabla de cotejo elaborada para establecer si en el Diseño Curricular de la Licenciatura de Psicología, se encuentra incluida la Política Ambiental de la USAC, se incluyen los siguientes criterios: a) ¿En el diseño curricular está contemplada la Política Ambiental?, b) ¿En el diseño curricular se encuentra la visión de la Política Ambiental de la USAC?, c) ¿En el diseño curricular se encuentra la misión de la Política Ambiental de la USAC?, d) ¿En el diseño curricular se contempla el perfil de los docentes relacionado con la Política Ambiental de la USAC?, e) $¿$ ¿n el diseño curricular se contempla el perfil de egreso del estudiante formado en Política Ambiental?.

Al analizar los criterios de la tabla de cotejo se establece con relación al diseño curricular lo siguiente:

a) Si está contemplada la Política Ambiental de la USAC, se establece que ésta no se menciona dentro de las políticas a desarrollarse en el Centro Universitario, únicamente se menciona que En el planeta se encuentran ciertos factores ambientales alterados los cuales presentan daños irreversibles y dentro de los cursos obligatorios no existe ninguno relacionado con la Política Ambiental.

b) Si incluye la visión de la Política Ambiental de la USAC, únicamente se señala que el Centro Universitario se compromete con los avances científico-tecnológico, social, humanista y ambiental de la región. 
c) Si se encuentra la misión de la Política Ambiental de la USAC, ésta no se contempla.

d) Si se contempla el perfil de los docentes relacionado con la Política Ambiental de la USAC y si se incluye en el perfil de egreso del estudiante en su formación la Política Ambiental, se concluye que en el diseño curricular no se toman en cuenta ninguno de los dos criterios.

Los resultados antes descritos para el análisis del Diseño Curricular, se alcanzaron utilizando una tabla de cotejo, la cual se presenta a continuación:

\section{Tabla 1}

Análisis de documento -Diseño Curricular de la Licenciatura de Psicología-.

Tabla de cotejo

\begin{tabular}{|c|c|c|c|}
\hline CRITERIOS & SI & NO & OBSERVACIONES \\
\hline $\begin{array}{l}\text { ¿En el diseño curricular está } \\
\text { contemplada la Política Ambiental de } \\
\text { la USAC? }\end{array}$ & & $x$ & $\begin{array}{l}\text { La política Ambiental de la USAC, no se menciona dentro de } \\
\text { las políticas a desarrollarse dentro del Centro Universitario. } \\
\text { Así mismo se menciona que las necesidades y demandas } \\
\text { de educación superior según el estudio realizado en el } \\
\text { Departamento se citan entre otras la Licenciatura en } \\
\text { Psicología y Licenciatura en Ecoturismo. Y en relación al } \\
\text { Plan Estratégico } 2000 \text { - 2022, solamente se menciona que. } \\
\text { En el planeta se encuentran ciertos factores ambientales } \\
\text { alterados los cuales presentan daños irreversibles. Dentro } \\
\text { de los cursos obligatorios no existe ninguno relacionado con } \\
\text { la Política Ambiental, sin embargo, sí existen los cursos de } \\
\text { Psicología Ambiental y Ecología y Sostenibilidad, pero éstos } \\
\text { pertenecen al área electiva. }\end{array}$ \\
\hline $\begin{array}{l}\text { ¿En el diseño curricular se encuentra } \\
\text { la visión de la Política Ambiental de } \\
\text { la USAC? }\end{array}$ & & $x$ & $\begin{array}{l}\text { La visión únicamente señala que el Centro Universitario se } \\
\text { compromete con los avances científico-tecnológico, social, } \\
\text { humanista y ambiental de la región. }\end{array}$ \\
\hline $\begin{array}{l}\text { ¿En el diseño curricular se encuentra } \\
\text { la misión de la Política Ambiental de } \\
\text { la USAC? }\end{array}$ & & $x$ & $\begin{array}{l}\text { La Misión del Centro Universitario no menciona la Política } \\
\text { Ambiental de la USAC. }\end{array}$ \\
\hline $\begin{array}{l}\text { ¿En el diseño curricular se contempla } \\
\text { el perfil de los docentes relacionado } \\
\text { con la Política Ambiental? }\end{array}$ & & $x$ & \\
\hline $\begin{array}{l}\text { ¿En el diseño curricular se contempla } \\
\text { el perfil de egreso del estudiante } \\
\text { formado en Política Ambiental? }\end{array}$ & & $\mathbf{x}$ & \\
\hline
\end{tabular}

Fuente: Análisis del Diseño Curricular de la Licenciatura en Psicología. (2020). 
Al tener a la vista cada uno de los criterios formulados para el análisis del diseño curricular de la licenciatura en Psicologia del CUNSAC, se pudo establecer que la política ambiental de la USAC está contemplada de manera muy superficial y que la misma no se encuentra en la visión, misión, así como tampoco se contempla el perfil de los docentes relacionados con dicha política y refleja también que no se contempla en el perfil de egreso del estudiante en esa casa de estudios.

\section{Discusión}

Del análisis del Diseño Curricular se establece que en la Licenciatura de Psicología no se contempla la Política Ambiental de la USAC, no obstante que la misma fue autorizada por el Consejo Superior Universitario, desde el año 2014. En la actualidad es preocupación de todos los centros educativos la implementación de la educación ambiental y la universidad no puede quedarse a la deriva. Según la Política Ambiental de la USAC, se debe observar y trabajar sobre el medio ambiente en todos los niveles, desde pre grado, grado, maestrías y doctorados.

El concepto de educación ambiental es dinámico, cambia continuamente como lo hace el medio ambiente y la percepción que de él se tiene. La humanidad establece relaciones con su medio a través de los niveles socioculturales, políticos y económicos los cuales son básicos (Bedoy, 2000). La formación ambiental debe ir dirigida a todos los humanos, ya que es un problema planetario de importancia para todos. (Wood y Walton, 1990). Es por ello que los docentes deben estar en constante capacitación y actualizados sobre el tema de medio ambiente.

Es importante lograr que el hombre individual y las colectividades logren comprender la naturaleza compleja e importante del ambiente natural y de lo que a la fecha ha creado el ser humano. que sea resultado de la interacción de sus aspectos biológicos, físicos, sociales, económicos y culturales; y alcancen las competencias y los valores, que les permitan participar de forma responsable y eficaz en la prevención y solucionar los problemas ambientales, y obtener una óptima gestión de la calidad ambiental; (Caduto, 1992). De allí que sea de vital importancia incluir en el diseño curricular los cursos que le provean a los futuros psicólogos las competencias en materia de medio ambiente que deben alcanzar al culminar su formación profesional, a fin de que, esos conocimientos, valores, comportamientos y habilidades prácticas, los puedan proyector en su comunidad. 
La educación ambiental va más allá del estudio de relaciones pedagógicas y ecológicas; lo principal es que el sistema educativo formal preparé a los educandos, para que a futuro puedan realizar los cambios necesarios para alcanzar un desarrollo sustentable: de igual manera crear conciencia para solucionar los problemas socioambientales que se enfrentan en la actualidad. (Caride, 2000). El objetivo de la educación ambiental debe apuntar a obtener cambio en la calidad de vida, en la conducta de los individuos y en las relaciones humanas, las cuales deben estar unidas a la solidaridad y el cuidado de todas las formas de vida y el planeta. (Febres y Florián, 2002). La pandemia del Covid-19 ha logrado que se privilegie el medio ambiente, ha creado conciencia de la relación con la naturaleza y la solidaridad que debe existir entre los habitantes del planeta.

La Política Ambiental de la USAC es integral, lo que hace falta es una férrea voluntad de ponerla en práctica para el bien de toda la comunidad y aportar soluciones a un problema planetario.

\section{Agradecimientos}

A los catedráticos de la Escuela de Estudios de Postgrado de la Facultad de Humanidades - USAC, a la Dra. Walda Flores Luin y al maestro Francisco Ureta, por brindarme su valioso acompañamiento, por asesorar y revisar el trabajo de investigación.

\section{Referencias bibliográficas}

Bedoy, V. (2000). La historia de la educación ambiental: reflexiones pedagógicas. En Educar REVISTA DE EDUCACION/NUEVA ÉPOCA NÚM. 13/ABRIL-JUNIO 2000.

Caduto, M. (1992). Guía para la enseñanza de valores ambientales. Programa Internacional de Educación Ambiental. Madrid, España: UNESCO-PNUMA.

Caride, J.A. (2002). Educación ambiental y desarrollo humano: Nuevas perspectivas conceptuales y estratégicas. Conferencia dictada en el III Congreso Iberoamericano de Educación Ambiental. Caracas, Venezuela. 
Durán, D. (2002). Escuela, ambiente y comunidad. Manual de capacitación docente. Integración del aprendizaje-servicio y la educación ambiental. Fundación Educambiente. Buenos Aires, Argentina: Programa Escuelas Solidarias.

Febres-Cordero, M. E. \& Florián, D. (2002). Políticas de educación ambiental y formación de capacidades para el desarrollo sustentable. De Río a Johannesburgo, La transición hacia el desarrollo sustentable. Seminario organizado por el PNUMA/ INE-SEMARNAT/ Universidad Autónoma Matropolitana.

PolíticaAmbientaldelaUniversidaddeSanCarlosdeGuatemalaaprobada por el Consejo Superior Universitario en sesión celebrada el 30 de julio de 2014 Punto Sexto, Inciso 6.2 Acta 13-2014.

Wood, D. \& Walton, D. (1990). Como planificar un programa de educación ambiental. (Cuadernos de la FAO). Washington D.C. USA: HED y UFWS.

\section{Sobre la autora}

Estudiante de la Maestría en Docencia Universitaria de la Universidad de San Carlos de Guatemala.

\section{Copyright (c) Irma Rosario Estrada Ortiz}

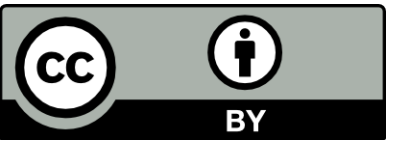

Este texto está protegido por una licencia Creative Commons 4.0.

Usted es libre para compartir, copiar y redistribuir el material en cualquier medio o formato y adaptar el documento, remezclar, transformar y crear a partir del material para cualquier propósito, incluso comercialmente, siempre que cumpla la condición de atribución: usted debe reconocer el crédito de una obra de manera adecuada, proporcionar un enlace a la licencia, e indicar si se han realizado cambios. Puede hacerlo en cualquier forma razonable, pero no de forma tal que sugiera que tiene el apoyo del licenciante o lo recibe por el uso que hace. 